\title{
Stability Analysis of Unified Chaotic System
}

\author{
Saad Fawzi AL-Azzawi \\ saad_fawzi78@yahoo.com
}

Department of Mathematics, College of Computer Sciences and

Mathematics, University of Mosul, Mosul, Iraq

Received on: 8/3/2009

Accepted on:17/5/2009

\begin{abstract}
The aim of this paper is to find the stability of unified chaotic system through studying different systems. The unified chaotic system is divided into three systems by depending on the values of $\theta$, when $0 \leq \theta<0.8$, the unified chaotic system becomes Lorenz system; when $\theta=0.8$; it becomes L $\ddot{u}$ system, when $0.8<\theta \leq 1$; the unified chaotic system becomes Chen system. Investigations the stability analysis of these systems leads to the stability of the unified chaotic system.
\end{abstract}

Keywords: unified chaotic system, Lorenz family, stability, Routh-Hurwitz

$$
\begin{aligned}
& \text { تحليل استقرارية النظام المضطرب الموحد } \\
& \text { سعد فوزي العزاوي } \\
& \text { كلبة علوم الحاسوب والرياضيات، جامعة الموصل } \\
& \text { تاريخ القبول: 2009/05/17 } \\
& \text { تاريخ الاستلام: 2009/03/08 } \\
& \text { الملخص } \\
& \text { الهدف من هذا البحث هو إيجاد استقرارية النظام المضطرب الموحد من خلال دراسة أنظمة } \\
& \text { مختلفة, حيث ينقسم النظام الموحد إلى ثلاثة أنظمة بالاعتماد على قيم } \theta \text { ر , بحيث عندما تكون }
\end{aligned}
$$

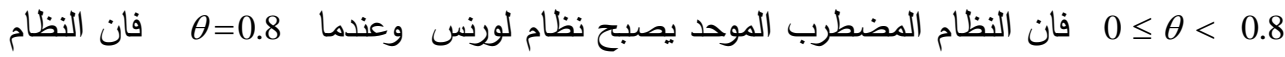

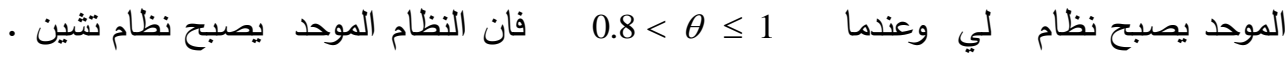

$$
\begin{aligned}
& \text { وبدراسة تحليل استقرارية هذه الأنظمة يقودنا إلى استقرارية النظام المضطرب الموحد. }
\end{aligned}
$$

الكلمات المفتاحية: النظام المضطرب الموحد, عائلة لور انس , الاستقرارية , معايير-Routh

Hurwitz

\section{1- Introduction:}

Over the last three decades, Chaos as very interesting nonlinear phenomena has been widely studied in various scientific fields. Recently, there has been increasing interest in controlling and utilizing chaos, particularly within the physicists and mathematicians and engineering and technological communities [5]. 
In 1963, Lorenz found the first chaotic system, which is a third order autonomous system with only two multiplication-type quadratic terms but displays very complex dynamical behaviors [10]. In 1999, Chen found another similar but topologically non-equivalent chaotic system, Chen system[3]. In 2002, L $\ddot{u}$ and Chen found a new chaotic system, bearing the name of the L $\ddot{u}$ system [1]. In the same year, L $\ddot{u}$ et al. unified above the three chaotic systems into one chaotic system which is called unified chaotic system [2].

The unified chaotic system was produced as a kind of unique and unified classification between the Lorenz and the Chen attractors, both in theory and in simulation. Of particularly interesting is that this unified system is likely the simplest chaotic system that bridges the gap between the Lorenz and the Chen system, and so it contributes to a better understanding of a family of 3dimensional autonomous quadratic chaotic systems [4].

The nonlinear differential equations that describe the unified chaotic system are following form $[2,4,8]$ :

$$
\left.\begin{array}{l}
\dot{x}=(25 \theta+10)(y-x) \\
\dot{y}=(28-35 \theta) x-x z+(29 \theta-1) y \\
\dot{z}=x y-\frac{\theta+8}{3} z
\end{array}\right\}
$$

Where $\theta \in[0,1]$, this system which describes a large family of chaotic systems containing the Lorenz and Chen system as two extremes and the $\mathrm{L} \ddot{u}$ system as a transition in between[10]. Recently, there are some analytical results reported about these chaotic systems, which are called the Lorenz family [3].

When $\theta \in[0,0.8)$, system (1) is called the generalized Lorenz system; when $\theta=0.8$; it becomes the generalized L $\ddot{u}$ system; when $\theta \in(0.8,1]$; system (1) is called the generalized Chen system [2,9].

The three critical points of system (1) can be found in [4] as follows:

$$
\begin{aligned}
& S_{0}(0,0,0) \\
& S_{-}(-\sqrt{(8+\sigma)(9-2 \sigma)},-\sqrt{(8+\sigma)(9-2 \sigma)} \quad, 27-6 \sigma \quad) \\
& S_{+}(\sqrt{(8+\sigma)(9-2 \sigma)}, \sqrt{(8+\sigma)(9-2 \sigma)} \quad, 27-6 \sigma \quad) \text {, }
\end{aligned}
$$

and studied the stability at these points. Finally, cleared that this system is unstable for $\theta \in[0,1]$, without depended on the Lorenz, Chen and L $\ddot{u}$ systems.

In this paper, we find the stability conditions of L $\ddot{u}$ system and obtain on helping results; finally, we find the same results which found in [4], but with 
another method, by using the three chaotic systems to investigate the stability of system (1).

\section{2- Preliminaries:}

The following notations will be used for the remaining of this paper.

The mathematical model of Lorenz system is a system of nonlinear ordinary differential equations which has the following form:

$$
\left.\begin{array}{l}
\dot{x}=\sigma(y-x) \\
\dot{y}=r x-x z-y \\
\dot{z}=x y-\beta z
\end{array}\right\}
$$

where $\sigma, r, \beta>o$, and Lorenz studied this system when $\sigma=10, r=28$,

$$
\beta=8 / 3,[6] \text {. }
$$

Chen system it also has the form of nonlinear ordinary differential equations:

$$
\left.\begin{array}{l}
\dot{x}=\sigma(y-x) \\
\dot{y}=(r-\sigma) x-x z+r y \\
\dot{z}=x y-\beta z
\end{array}\right\}
$$

where $\sigma, r, \beta>o$ and $\sigma>r$, Chen take $\sigma=35, r=28, \beta=3$ [5].

The mathematical model of $\mathbf{L} \ddot{u}$ system also is a system of nonlinear ordinary differential equations which has the form:

$$
\left.\begin{array}{l}
\dot{x}=\sigma(y-x) \\
\dot{y}=-x z+r y \\
\dot{z}=x y-\beta z
\end{array}\right\}
$$

also $\sigma, r, \beta>0, \sigma>r, \mathrm{~L} \ddot{u}$ studied this system when $\sigma=36, r=20, \beta=3$ [1].

One can see that the three dynamical systems, all are nonlinear ordinary differential equation systems of third degree. And all $\mathrm{x}$ derivative in those systems are multiplied by a number $\sigma$. The only difference is the form of y derivative [1]. According to Vanecek and Celikovsky definition. They separated the system into linear and quadratic parts. In the linear part of system described by the matrix $A=\left(a_{i j}\right)_{3 \times 3}$, the Lorenz system satisfies the condition $a_{12} a_{21}>0$ while the Chen system satisfies $a_{12} a_{21}<0$ and L $\ddot{u}$ system satisfies the condition $a_{12} a_{21}=0 \quad[8,9]$. 


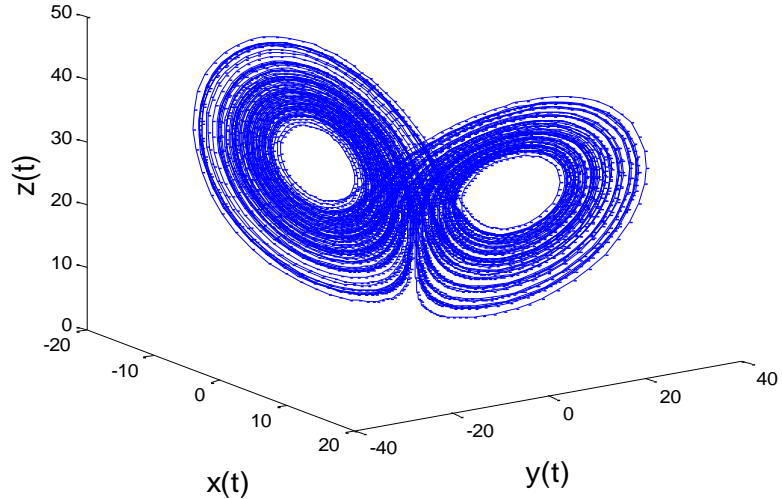

Figure 1: the attractor of Lorenz system

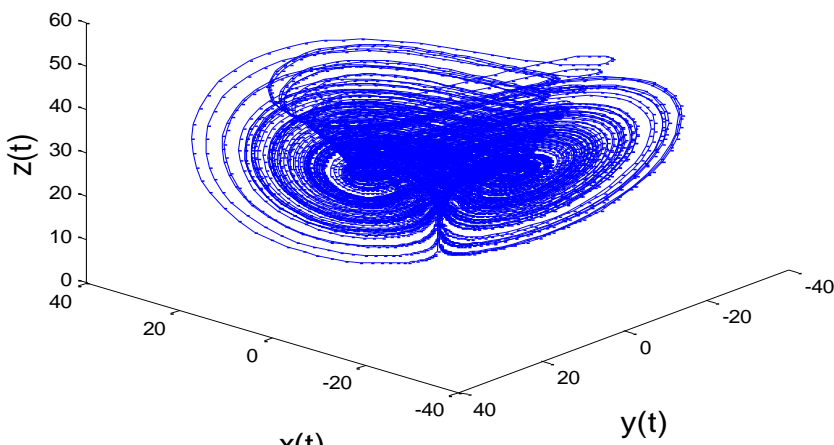

Figure 2: the attractor of $\mathrm{L} \ddot{u}$ system

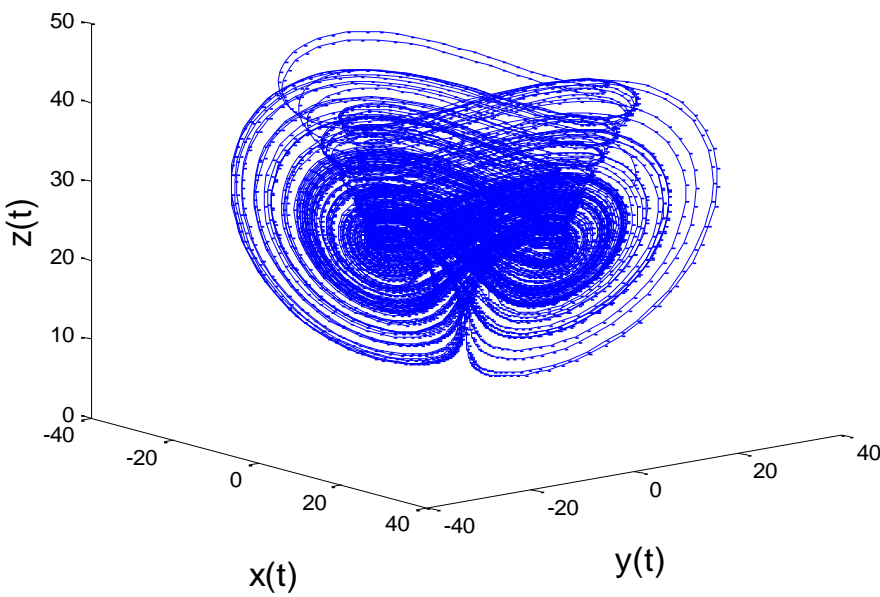

Figure 3: the attractor of Chen system 


\section{3- Helping Results:}

Regarding the basic dynamical behaviors of the Lorenz system, we have the following observations.

Lemma 1 [7]: In system (2)

(i) if $\mathrm{r}<1$, then this system has only one critical point, $S_{0}(0,0,0)$;

(ii ) if $\mathrm{r}>1$, then this system has three critical points : $S_{0}(0,0,0)$

$S_{+}\left(x_{0}, y_{0}, z_{0}\right), S_{-}\left(-x_{0},-y_{0}, z_{0}\right)$, Where $x_{0}=y_{0}=\sqrt{\beta(r-1)}$ and

$z_{0}=r-1$.

In fact, linearized system (2) about the critical point $S_{0}(0,0,0)$

yields the following characteristic equation [7]:

$f(\lambda)=\lambda^{3}+(\sigma+1+\beta) \lambda^{2}+[\beta(\sigma+1)+\sigma(1-r)] \lambda+\beta \sigma(1-r)=0$

While the linearized system (2) about the critical point $S_{+}$or $S_{-}$yields the following characteristic equation [7]:

$f(\lambda)=\lambda^{3}+(\sigma+1+\beta) \lambda^{2}+\beta(\sigma+r) \lambda+2 \beta \sigma(r-1)=0$

Theorem 1 [8]: In system (2)

(i) if $\mathrm{r}<1$, then the critical point $S_{0}(0,0,0)$ is asymptotically stable ;

(ii) if $\mathrm{r}>1$, then the critical point $S_{0}(0,0,0)$ is unstable ;

(iii) if $r<r_{c}=\frac{\sigma(\sigma+\beta+3)}{\sigma-\beta-1}$, then the critical points $S_{+}$and $S_{-}$are all asymptotically stable ;

(iiii) if $r>r_{c}$, then the critical points $S_{+}$and $S_{-}$are all unstable .

Now, the basic dynamical behaviors of the Chen system is included in the following lemma.

Lemma 2 [5]: In system (3)

(i) if $\sigma>2 r$, then this system has only one critical point, $S_{0}(0,0,0)$;

(ii) if $\sigma<2 r$, then this system has three critical points : $S_{0}(0,0,0)$

$S_{+}\left(x_{0}, y_{0}, z_{0}\right), S_{-}\left(-x_{0},-y_{0}, z_{0}\right)$, where $\quad x_{0}=y_{0}=\sqrt{\beta(2 r-\sigma)} \quad$ and

$z_{0}=2 r-\sigma$.

Linearized system (3) about the critical points $S_{0}$ and $S_{+}$or $S_{-}$yields the following characteristic equations respectively [5]:

$f(\lambda)=\lambda^{3}+(\sigma+\beta-r) \lambda^{2}+[\beta(\sigma-r)+\sigma(\sigma-2 r)] \lambda+\beta \sigma(\sigma-2 r)=0 \ldots(7)$

$f(\lambda)=\lambda^{3}+(\sigma+\beta-r) \lambda^{2}+\beta r \lambda+2 \beta \sigma(2 r-\sigma)=0$

Theorem 2 [5]: In system (3)

(i) if $\sigma>2 r$, then the critical point $S_{0}(0,0,0)$ is asymptotically stable;

(ii) if $\sigma<2 r$, then the critical point $S_{0}(0,0,0)$ is unstable ; 
(iii) if $\sigma<2 r$ and $(\sigma+\beta-r) r-2 \sigma(2 r-\sigma)>0$, then the critical points $S_{+}$and $S_{-}$are all stable.

\section{4- Main results:}

It is easy to verify that the $\mathrm{L} \ddot{u}$ system has three critical points: $S_{0}(0,0,0), S_{+}\left(x_{0}, y_{0}, z_{0}\right), S_{-}\left(-x_{0},-y_{0}, z_{0}\right)$, where $\quad x_{0}=y_{0}=\sqrt{\beta r}$ and $z_{0}=r$

Proposition 1: The critical point $S_{0}(0,0,0)$ is always unstable.

Proof: At the critical point $S_{0}(0,0,0)$, is linearized, the Jacobian matrix of system (4) is defined as ;

$$
J_{S_{0}}=\left[\begin{array}{ccc}
-\sigma & \sigma & 0 \\
0 & r & 0 \\
0 & 0 & -\beta
\end{array}\right]
$$

The characteristic equation is:

$$
\begin{aligned}
& f(\lambda)=\lambda^{3}+(\sigma+\beta-r) \lambda^{2}+[\beta(\sigma-r)-\sigma r] \lambda-\beta \sigma r=0 \\
& \text { Or } \quad(\lambda+\beta) \cdot\left(\lambda^{2}+(\sigma-r) \lambda-\sigma r\right)=0
\end{aligned}
$$

either $\quad(\lambda+\beta)=0$ or $\lambda^{2}+(\sigma-r) \lambda-\sigma r=0$, and three eigenvalues corresponding to the critical point $S_{0}$ are:

$$
\lambda_{1}=-\beta, \lambda_{2}=-\sigma, \quad \lambda_{3}=r
$$

So, it's clear that the parameter $\mathrm{r}>0$, therefore $\lambda_{3}>0$, consequently the critical point $S_{0}(0,0,0)$ is always unstable. This completes the proof.

In the following, we consider the stability of the system (4) at the critical points $S_{+}$and $S_{-}$, Because the system is invariant under the transformation[1], so one only needs to consider the stability of any one of the both . The stability of the system (4) at critical point $S_{+}$is analyzed in this paper.

Under the linear transformation $(x, y, z) \rightarrow(X, Y, Z)$ :

$$
\left.\begin{array}{l}
x=X+\sqrt{\beta r} \\
y=Y+\sqrt{\beta r} \\
z=Z+r
\end{array}\right\}
$$

the system (4) becomes 


$$
\left.\begin{array}{l}
\dot{X}=\sigma(Y-X) \\
\dot{Y}=-r X+r Y-\sqrt{\beta r} Z \\
\dot{Z}=\sqrt{\beta r} X+\sqrt{\beta r} Y-\beta Z
\end{array}\right\}
$$

The critical point $S_{+}$of the system (4) is swiched to the new critical point $S_{0}^{\prime}(0,0,0)$ of the system (13) under the linear transformation, in the following, the stability of system (13) at the critical point $S_{0}^{\prime}$ is considered.

The Jacobian matrix of the system (13) at $S_{0}^{\prime}(0,0,0)$ is :

$$
J\left(S_{0}^{\prime}\right)=\left[\begin{array}{ccc}
-\sigma & \sigma & 0 \\
-r & r & -\sqrt{\beta r} \\
\sqrt{\beta r} & \sqrt{\beta r} & -\beta
\end{array}\right]
$$

and the characteristic equation is :

$$
f(\lambda)=\lambda^{3}+(\sigma+\beta-r) \lambda^{2}+\beta \sigma \lambda+2 \beta \sigma r=0 .
$$

Theorem 3: In the system (4)

(i) if $\sigma+\beta>3 r$, then the critical point $S_{+}$is asymptotically stable ;

(ii) if $\sigma+\beta<3 r$, then the critical point $S_{+}$is unstable .

Proof: From the equation (15), Let

$$
\left.\begin{array}{l}
A=\sigma+\beta-r \\
B=\beta \sigma \\
C=2 \beta \sigma r
\end{array}\right\}
$$

Then the Routh-Hurwitz conditions lead to the conclusion that the real parts of the roots are negative if and only if $A>0, C>0$ and $A B>C$.

$\sigma+\beta-r>0$ always, since $\sigma>r$ and clear that $2 \beta \sigma r>0$, consequently we must prove that $(\sigma+\beta-r) \beta \sigma>2 \beta \sigma r$ $(\sigma+\beta-r) \beta \sigma>2 \beta \sigma r \Rightarrow \sigma+\beta-r>2 r$

$\therefore$ when $\sigma+\beta>3 r$ Then the critical point $S_{+}$is asymptotically stable and when $\sigma+\beta<3 r$ then the critical point $S_{+}$is unstable. This completes the proof.

Now, to simplify computations for our results, we described the system (1) as follows:

$$
\left.\begin{array}{l}
\dot{x}=\sigma_{1}(y-x) \\
\dot{y}=r_{1} x-x z+r_{2} y \\
\dot{z}=x y-\beta_{1} z
\end{array}\right\}
$$

Where $\sigma_{1}=25 \theta+10, r_{1}=28-35 \theta, r_{2}=29 \theta-1, \beta_{1}=\frac{\theta+8}{3}$. 
The system (17) has three critical points which are $S_{0}(0,0,0)$, $S_{+}\left(x_{0}, y_{0}, z_{0}\right), S_{-}\left(-x_{0},-y_{0}, z_{0}\right)$, where $x_{0}=y_{0}=\sqrt{\beta\left(r_{1}+r_{2}\right)}$ and $z_{0}=r_{1}+r_{2}$.

The Jacobian matrix of system (17) at the critical point $S_{0}$ is defined as:

$$
J_{S_{0}}=\left[\begin{array}{ccc}
-\sigma_{1} & \sigma_{1} & 0 \\
r_{1} & r_{2} & 0 \\
0 & 0 & -\beta_{1}
\end{array}\right]
$$

and the characteristic equation is

$$
f(\lambda)=\lambda^{3}+\left(\sigma_{1}+\beta_{1}-r_{2}\right) \lambda^{2}+\left[\beta_{1}\left(\sigma_{1}-r_{2}\right)-\sigma_{1}\left(r_{1}+r_{2}\right)\right] \lambda+\beta_{1} \sigma_{1}\left(-r_{1}-r_{2}\right)=0
$$

We will use the following Corollary, which enables us to find the Jacobian matrix directly for Lorenz family at $S_{+}$and $S_{-}$without using transformation.

\section{Corollary1:}

Let $D=\left[\begin{array}{lll}d_{11} & d_{12} & d_{13} \\ d_{21} & d_{22} & d_{23} \\ d_{31} & d_{32} & d_{33}\end{array}\right]$ is a Jacobian matrix at $S_{0}$, then $E=\left[\begin{array}{lll}e_{11} & e_{12} & e_{13} \\ e_{21} & e_{22} & e_{23} \\ e_{31} & e_{32} & e_{33}\end{array}\right]$ is a Jacobian matrix at $S_{+}$,

$$
\text { where } \begin{aligned}
e_{11} & =d_{11} \\
e_{12} & =d_{12} \\
e_{13} & =d_{13}
\end{aligned} \text {, and } \begin{aligned}
e_{21} & =-d_{22} \\
e_{22} & =d_{22} \\
e_{23} & =-x_{0}
\end{aligned}, \text { and } \begin{aligned}
e_{31} & =x_{0} \\
e_{32} & =x_{0} \\
e_{33} & =d_{33}
\end{aligned}
$$

So, the Jacobian matrix at $S_{+}$by using Corollary 1 is given by:

$$
J\left(S_{0}^{\prime}\right)=\left[\begin{array}{ccc}
-\sigma_{1} & \sigma_{1} & 0 \\
-r_{2} & r_{2} & -\sqrt{\beta_{1}\left(r_{1}+r_{2}\right)} \\
\sqrt{\beta_{1}\left(r_{1}+r_{2}\right)} & \sqrt{\beta_{1}\left(r_{1}+r_{2}\right)} & -\beta_{1}
\end{array}\right]
$$

and the characteristic equation is :

$$
f(\lambda)=\lambda^{3}+\left(\sigma_{1}+\beta_{1}-r_{2}\right) \lambda^{2}+\beta_{1}\left(\sigma_{1}+r_{1}\right) \lambda+2 \beta_{1} \sigma_{1}\left(r_{1}+r_{2}\right)=0
$$

\section{Corollary2:}

The third coefficient values ( $\mathrm{C}$ ) of the cubic equations $(5,6,7,8,10,15,19,21)$ depended on the third value of $S_{+} \quad\left(z_{0}\right)$ as following : 


$$
\text { and } \quad \begin{aligned}
& C_{5}=C_{7}=C_{10}=C_{19}=-\beta \sigma\left(z_{0}\right) \\
& C_{6}=C_{8}=C_{15}=C_{21}=2 \beta \sigma\left(z_{0}\right) .
\end{aligned}
$$

Theorem 4: In system (17)

(i) The critical point $S_{0}$ is unstable if one of the following cases holds:

$10 \leq \theta<0.8$ and $r_{1}>1$;

$2 \theta=0.8$ for any value of $\sigma_{1}, r_{1}, r_{2}, \beta_{1}$;

$3 \quad o .8<\theta \leq 1$ and $\sigma_{1}<2 r_{1}$.

(ii) The critical point $S_{+}$is unstable if one of the following cases holds:

$10 \leq \theta<0.8$ and $r_{1}>r_{c}=\frac{\sigma_{1}\left(-\sigma_{1}-\beta_{1}+3 r_{1}\right)}{-\sigma_{1}+\beta_{1}-r_{2}}$;

$2 \theta=0.8 \quad$ and $\quad \beta_{1}+\sigma_{1}<3 r_{1}$;

$3 o .8<\theta \leq 1$ for any value of $\sigma_{1}, r_{1}, r_{2}, \beta_{1}$,

Proof:

(i)(1) when $\theta \in[0,0.8)$,the system (17) is the generalized system(2), and $r_{1} \cong r$, since $r_{1}>1$ (given), then $\mathrm{r}>1$ also, the system (2) is unstable (Ttheorem 1, case(ii)), therefore system (17) is unstable at $S_{0}$.

(2) when $\theta=0.8$,system (17) becomes the generalized system(4), and by Proposition 1,then system(4) is always unstable, consequently system (17) is unstable at $S_{0}$.

(3) when $\theta \in(0.8,1]$, system (17) belongs to the generalized system(3), and $\sigma_{1} \cong \sigma, r_{1} \cong r$, since $\sigma_{1}<2 r_{1}$ (given), then $\sigma<2 r$ and satisfied theorem 2 case (ii) ,so system(3) is unstable, therefore system (17) is unstable also at $S_{0}$.

(ii)(1) when $\theta \in[0,0.8)$, system (17) is the generalized system(2), and $r_{1} \cong r$, since $r_{1}>r_{c}=\frac{\sigma_{1}\left(-\sigma_{1}-\beta_{1}+3 r_{1}\right)}{-\sigma_{1}+\beta_{1}-r_{2}}$ (given), then $r>\frac{\sigma(\sigma+\beta+3)}{\sigma-\beta-1}$ also , so system (2) is unstable (Ttheorem 1 ,case(iiii)), therefore system (17) is unstable at $S_{+}$.

(2) when $\theta=0.8$,system (17) becomes the generalized system(4),

and $\sigma_{1} \cong \sigma, r_{1} \cong r, \beta_{1} \cong \beta$, and $\beta_{1}+\sigma_{1}<3 r_{1}$ (given) then $\beta+\sigma<3 r$, so satisfied theorem 3 case (ii), hence $\operatorname{system}(4)$ is

unstable, consequently system (17) is unstable also at $S_{+}$.

(3) when $\theta \in(0.8,1]$, system (17) belongs to the generalized system(3), and from equation 8, we have $A>0, C>0$ but $A B<C$, not satisfy Routh-Hurwitz criterion hence the real part of at least one root is positive, therefore system(3) is unstable, consequently system (17) is unstable also at $S_{+}$. 


\section{5 - Illustrative Example:}

Example: Investigate for stability of system 1

Solution: if $\theta=0$, then system (1)becomes system(2)

$$
\left.\begin{array}{rl}
\dot{x} & =10(y-x) \\
\dot{y} & =28 x-x z-y \\
\dot{z} & =x y-\frac{8}{3} z
\end{array}\right\}
$$

By theorem (4), case (i) first condition, the system (22) is unstable at $S_{0}$ since $\mathrm{r}=28>1$ and unstable at $S_{+}$since $\mathrm{r}=28$ and $r_{c}=-85.6$ so $r>r_{c}$.

if $\theta=1$, then system (1)becomes system (3)

$$
\left.\begin{array}{l}
\dot{x}=35(y-x) \\
\dot{y}=(28-35) x-x z+28 y \\
\dot{z}=x y-3 z
\end{array}\right\}
$$

Since $\sigma=35,2 r=56$ hence $\sigma<2 r$ therefore satisfied theorem (4), case (i) third condition therefore system (23) is unstable at $S_{0}$ and also unstable at $S_{+}$by the same theorem, case (ii) third condition.

if $\theta=0.8$, then system (1)becomes system (4)

$$
\left.\begin{array}{l}
\dot{x}=30(y-x) \\
\dot{y}=-x z+23.2 y \\
\dot{z}=x y-\frac{8.8}{3} z
\end{array}\right\}
$$

By theorem (4), case ( $\mathrm{i}$ ) second condition, the system (24) is unstable at $S_{0}$ and unstable at $S_{+}$since $\beta+\sigma=32.93$ while $3 r=69.9$, hence $\beta+\sigma<3 r$ therefore satisfied theorem (4), case (ii) third condition.

\section{6 - Conclusion:}

In this paper, we have investigated the stability of unified chaotic system by using three chaotic systems (Lorenz, Chen and L $\ddot{u}$ systems). By this method we justified the same results which have been found by previous methods. An illustrative example shows the effectiveness and feasibility of this method. 


\section{REFERENCES}

[1] Cai G. , Tian L. , Fan X., (2006), Slow Manifold Model and simulation of the $\mathrm{L} \ddot{u}$ System, Journal of Information and Computing Science, Vol. 1 , No. 2 , pp. $78-84$.

[2] Cai G. , Tu W. , (2007), Adaptive Backstopping Control of the Uncertain Unified Chaotic System, International Journal of Nonlinear Science, Vol. 4 , No. 1, pp. 17 - 24 .

[3] Li D. , Lu J. , Wu X. , Chen G. ,(2005) , Estimating the Bounds for the pp. $529-534$.

[4] L ü J. , Chen G. , Yu X. , Zhany S. , (2003), Dynamical Behaviors of a Unified Chaotic Systems, Intelligent and Complex Systems, DCDIS Proceedings1, pp. 115 - 124.

[5] L $\ddot{u}$ J. , Zhou T. , Chen G. ,Zhang S. , (2002),Local Bifurcations of the Chen System, International Journal of Bifurcation and Chaos, Vol. 12, No. 10, pp. 2257- 2270.

[6] Teschi G. , (2009), Ordinary Differential equations and Dynamical Systems, AMS-LATEX AND Makeindex , Inc.

[7] Thanoon T. Y. , AL-Azzawi S. F. , Accepted on : 23/12/2008 ,Stability of Lorenz Differential System By Parameters, Tikrit Journal of Pure Science.

[8] Wu X. , Lu J. , Tse C. , Wany J. , Liu J. , (2007) , Impulsive Control and Synchronization of the Lorenz Systems Family, Chaos, Solitons and Fractals, Vol. 31, pp. 631-638.

[9] Xiang-Rong G. , Chong-Xin L .,Fa-Qiang W. , (2008), Circuit Realization of the Fractional- order Unified Chaotic System, Chin. Phys. Soc. And IOP Publishing Ltd, Vol. 17 , No. 5, pp. 1664-1669.

[10] Yong -Lu S. , Yong - hao Z., (2008), Estimating the Globally Attractive Set and Positively Invariant Set of a Unified Chaotic System, Journal of Chongqing University (English Edition), Vol. 7, No. 3, pp. $216-220$. 